\section{A combination treatment approach and cord blood stem cell transplant for blastic plasmacytoid dendritic cell neoplasm}

We read with great interest the article on blastic plasmacytoid dendritic cell neoplasm (BPDCN) by Pagano et al. ${ }^{1}$ The authors propose a strategy of combining ALL-like and AML-like protocols for this rare and aggressive disease. The authors also emphasize the importance of consolidating when possible with an allogeneic stem cell transplant, in light of the poor prognosis (median overall survival of 8.7 months) of BPDCN. We would like to share here our experience of successfully treating a patient with cord blood stem cell transplantation using a preparative regimen of thiotepa, fludarabine and melphalan. ${ }^{2}$

Our patient is a 39-year old female who presented with a history of easy bruising and shortness of breath. No skin lesion or lymph node enlargement was noted at the time of diagnosis. Peripheral blood examination showed pancytopenia. Absolute neutrophil count was $0.9 \times 10^{9} / \mathrm{L}$, hemoglobin $7.9 \mathrm{~g} / \mathrm{dL}$, platelet count $21 \times 10^{9} / \mathrm{L}$, and there was no evidence of blasts in the peripheral blood. A subsequent bone marrow aspirate and biopsy confirmed a diagnosis of $\mathrm{BPDCN}$. The blasts in the bone marrow were positive for CD2, CD4, CD7, CD8, CD56, CD64, HLA-DR by flow cytometry analysis and positive for CD123 and TCL $1^{3}$ by immunoperoxidase staining. The blasts were negative for CD34, TdT, CD13, CD33, CD10, as well as for lineage specific markers including CD20, CD19, cytoplasmic CD22 for B cells, surface CD3, cytoplasmic-CD3 for T cells, and myeloperoxidase for myeloid cells. Fluorescence in situ hybridization for EBV encoded RNA (EBER) was also negative. Although CD8 expression is not typical, the diagnosis of BPDCN was favored over aggressive NK-cell leukemia based on agranular cytoplasm of blasts and lack of EBV expression. Cytogenetic analysis showed a normal female karyotype with no cytogenetic aberrations. Cerebrospinal fluid (CSF) cytology showed no evidence of involvement by the malignant cells.

The patient was initially treated with a standard regimen for aggressive T/NK cell malignancies called "SMILE" that consisted of steroid (dexamethasone), methotrexate, ifosfamide, L-asparaginase and etoposide. ${ }^{4}$ Day 21 bone marrow biopsy after the first cycle showed a partial response (PR) based on a greater than $50 \%$ reduction in blast percentage (from $60 \%$ down to $20 \%$ ). She was subsequently treated with an AML regimen that consisted of high-dose cytarabine and mitoxantrone. ${ }^{5}$ She achieved a complete remission (CR) and was consolidated 11 weeks after diagnosis with a cord blood stem cell transplant. The preparative regimen consisted of thiotepa $(5 \mathrm{mg} / \mathrm{kg})$ Day -7 , fludarabine $\left(30 \mathrm{mg} / \mathrm{m}^{2}\right)$ Day -6 through Day -2 , melphalan $140 \mathrm{mg} / \mathrm{m}^{2}$ on Day -1 and rabbit ATG (3 $\mathrm{mg} / \mathrm{kg}) .^{6}$ A single cord blood unit with $3.81 \times 10^{7}$ total nucleated cells $/ \mathrm{kg}$ and $2.95 \times 10^{5} \mathrm{CD} 34$ cells $/ \mathrm{kg}$ was infused on Day 0 . The unit was 4 of 6 HLA matched with the patient based on HLA A, B and DR typing. Graft-versushost disease (GVHD) prophylaxis consisted of tacrolimus and mycophenolate mofetil. The patient engrafted her neutrophils on Day 18 and platelets on Day 39 post transplant. One hundred percent donor chimerism was achieved by Day 100 . She is currently at 313 days (10.4 months) since her diagnosis and 237 days (7.9 months) since her cord blood stem cell transplant. She is being followed as an outpatient on tapering doses of immunosup- pression. She is doing well except for mild grade 1-2 skin GVHD that has been responsive to topical steroids.

Due to the scarsity of data and lack of consensus as to the optimal treatment approach for BPDCN, we used an approach that combined ALL-like and AML-like treatment protocols in sequence and achieved a complete remission. Since our patient did not have a matched related or unrelated donor, she underwent a cord blood stem cell transplant. This is the first case report demonstrating the successful use of a cord blood stem cell donor in this rare and aggressive disease.

A sequential combination approach alternating ALLand AML-like induction regimens until CR followed by consolidation with a stem cell transplant when possible is an effective treatment strategy and warrants consideration in patients with this rare and unfavorable prognosis disease. Cord blood stem cell transplant in the absence of a matched related or unrelated donor is feasible and should be attempted in patients who are candidates for transplant. The advantages of using a cord blood stem cell donor include, among others, increased ability to find a donor and reduced incidence of life threatening grade 3 or 4 acute GVHD.

Muthalagu Ramanathan, Jan Cerny, Hongbo Yu, Bruce A. Woda, and Rajneesh Nath

University of Massachusetts Medical School and Medical Center, USA

Correspondence: muthalagu.ramanathan@umassmemorial.org doi:10.3324/haematol.2012.080051

Key-words: blastic plasmacytoid dendritic cell neoplasm, cord blood transplant

Information on authorship, contributions, and financial \& other disclosures was provided by the authors and is available with the online version of this article at www. haematologica.org.

\section{References}

1. Pagano L, Valentini CG, Pulsoni A, Fisogni S, Carluccio P, Mannelli F, et al. Blastic plasmacytoid dendritic cell neoplasm with leukemic presentation: an Italian multicenter study. Haematologica; 2013;98(2):239-46.

2. Ciurea SO, Saliba RM, Hamerschlak N, Karduss Aurueta AJ, Bassett $\mathrm{R}$, Fernandez-Vina M, et al. Fludarabine, melphalan, thiotepa and anti-thymocyte globulin conditioning for unrelated cord blood transplant. Leuk Lymphoma. 2012;53(5):901-6.

3. Herling M, Teitell MA, Shen RR, Medeiros LJ, Jones D. TCL1 expression in plasmacytoid dendritic cells (DC2s) and the related CD4+CD56+ blastic tumors of skin. Blood. 2003;101(12):5007-9.

4. Yamaguchi M, Kwong YL, Kim WS, Maeda Y, Hashimoto C, Suh C, et al. Phase II Study of SMILE Chemotherapy for Newly Diagnosed Stage IV, Relapsed, or Refractory Extranodal Natural Killer (NK)/TCell Lymphoma, Nasal Type: The NK-Cell Tumor Study Group Study. J Clin Oncol. 2011;29(33):4410-6.

5. Ramanathan M, Cerny J, Raffel G, Petrillo-Deluca L, Tarrasky N, Nath R. Increased Ability to Transplant and Improved Survival in Patients with High Risk Acute Myeloid Leukemia (AML) After Induction with High Dose Cytarabine and Mitoxantrone (HIDAC/MITO). Biol Blood Marrow Transplant. 2012;18(2 Suppl):S291-2

6. Nath R, Glen R, William W, Jan C, Ramanathan M, Petrillo-Deluca $\mathrm{L}$, et al. Successful Engraftment and Reduced Early Mortality in Unrelated Donor (UD) Cord Blood (CB) Stem Cell Transplantation (SCT) Using a Preparative Regimen of Thiotepa, Fludarabine, Melphalan and Rabbit Anti-Thymocyte Globulin (TFM/r-ATG) in Older Adults With a Single Unmanipulated CB Unit. Biol Blood Marrow Transplant. 2011;17(2 Suppl):S228-9.

7. Cohena Y, Nagler A. Hematopoietic stem-cell transplantation using umbilical-cord blood. Leuk Lymphoma. 2003;44(8):1287-99. 